\title{
Anhang 2a: Follower-Netzwerk (Alternative Nachrichtenmedien)
}

\begin{tabular}{|c|c|c|c|c|c|c|c|}
\hline Account & Modularity Class & $\begin{array}{c}\text { In- } \\
\text { degree }\end{array}$ & $\begin{array}{c}\text { Out- } \\
\text { degree }\end{array}$ & Degree & $\begin{array}{l}\text { Closeness } \\
\text { Centrality }\end{array}$ & $\begin{array}{l}\text { Betweenness } \\
\text { Centrality }\end{array}$ & $\begin{array}{c}\text { Eigen. } \\
\text { Centrality }\end{array}$ \\
\hline Der_Postillon & $\begin{array}{l}\text { Mainstream und } \\
\text { Alternativmedien } \\
\text { Typ IV }\end{array}$ & 11157 & 48 & 11205 & 0,318 & 18778739,236 & 0,789 \\
\hline tagesschau & $\begin{array}{l}\text { Mainstream und } \\
\text { Alternativmedien } \\
\text { Typ IV }\end{array}$ & 10583 & 35 & 10618 & 0,286 & 4666276,644 & 0,850 \\
\hline SZ & $\begin{array}{l}\text { Mainstream und } \\
\text { Alternativmedien } \\
\text { Typ IV }\end{array}$ & 8903 & 25 & 8928 & 0,287 & 1765506,801 & 0,749 \\
\hline netzpolitik & $\begin{array}{l}\text { Mainstream und } \\
\text { Alternativmedien } \\
\text { Typ IV }\end{array}$ & 7278 & 224 & 7502 & 0,331 & 6442331,736 & 0,608 \\
\hline politicalbeauty & $\begin{array}{l}\text { Mainstream und } \\
\text { Alternativmedien } \\
\text { Typ IV }\end{array}$ & 7014 & 844 & 7858 & 0,377 & 30803585,256 & 0,469 \\
\hline BILDblog & $\begin{array}{l}\text { Mainstream und } \\
\text { Alternativmedien } \\
\text { Typ IV }\end{array}$ & 6784 & 41 & 6825 & 0,296 & 3196415,617 & 0,496 \\
\hline welt & $\begin{array}{l}\text { Mainstream und } \\
\text { Alternativmedien } \\
\text { Typ IV }\end{array}$ & 6515 & 23 & 6538 & 0,294 & 1989932,650 & 0,731 \\
\hline Volksverpetzer & $\begin{array}{l}\text { Mainstream und } \\
\text { Alternativmedien } \\
\text { Typ IV }\end{array}$ & 6065 & 174 & 6239 & 0,343 & 14117582,956 & 0,337 \\
\hline TiloJung & $\begin{array}{l}\text { Mainstream und } \\
\text { Alternativmedien } \\
\text { Typ IV }\end{array}$ & 6014 & 161 & 6175 & 0,334 & 8028924,236 & 0,462 \\
\hline ZDFheute & $\begin{array}{l}\text { Mainstream und } \\
\text { Alternativmedien } \\
\text { Typ IV }\end{array}$ & 5852 & 31 & 5883 & 0,299 & 1972654,185 & 0,530 \\
\hline $\begin{array}{l}\text { Natascha_ } \\
\text { Strobl }\end{array}$ & $\begin{array}{l}\text { Mainstream und } \\
\text { Alternativmedien } \\
\text { Typ IV }\end{array}$ & 5653 & 881 & 6534 & 0,371 & 25700820,949 & 0,377 \\
\hline
\end{tabular}




\begin{tabular}{|c|c|c|c|c|c|c|c|}
\hline Account & Modularity Class & $\begin{array}{c}\text { In- } \\
\text { degree }\end{array}$ & $\begin{array}{c}\text { Out- } \\
\text { degree }\end{array}$ & Degree & $\begin{array}{l}\text { Closeness } \\
\text { Centrality } \\
\end{array}$ & $\begin{array}{c}\text { Betweenness } \\
\text { Centrality }\end{array}$ & $\begin{array}{c}\text { Eigen. } \\
\text { Centrality }\end{array}$ \\
\hline ZDF & $\begin{array}{l}\text { Mainstream und } \\
\text { Alternativmedien } \\
\text { Typ IV }\end{array}$ & 5602 & 33 & 5635 & 0,292 & 1340530,930 & 0,514 \\
\hline $\begin{array}{l}\text { FridayFor } \\
\text { Future }\end{array}$ & $\begin{array}{l}\text { Mainstream und } \\
\text { Alternativmedien } \\
\text { Typ IV }\end{array}$ & 5127 & 0 & 5127 & 0,000 & 0,000 & 0,313 \\
\hline dielinke & $\begin{array}{l}\text { Mainstream und } \\
\text { Alternativmedien } \\
\text { Typ IV }\end{array}$ & 4753 & 198 & 4951 & 0,342 & 7027357,209 & 0,413 \\
\hline correctiv_org & $\begin{array}{l}\text { Mainstream und } \\
\text { Alternativmedien } \\
\text { Typ IV }\end{array}$ & 4661 & 545 & 5206 & 0,369 & 13891565,077 & 0,428 \\
\hline ArminWolf & $\begin{array}{l}\text { Mainstream und } \\
\text { Alternativmedien } \\
\text { Typ IV }\end{array}$ & 4040 & 110 & 4150 & 0,313 & 1876566,466 & 0,356 \\
\hline chaosupdates & $\begin{array}{l}\text { Mainstream und } \\
\text { Alternativmedien } \\
\text { Typ IV }\end{array}$ & 4011 & 19 & 4030 & 0,285 & 811714,441 & 0,286 \\
\hline derfreitag & $\begin{array}{l}\text { Mainstream und } \\
\text { Alternativmedien } \\
\text { Typ IV }\end{array}$ & 3872 & 530 & 4402 & 0,367 & 10325196,840 & 0,377 \\
\hline lobbycontrol & $\begin{array}{l}\text { Mainstream und } \\
\text { Alternativmedien } \\
\text { Typ IV }\end{array}$ & 3800 & 106 & 3906 & 0,318 & 1312755,733 & 0,345 \\
\hline uebermedien & $\begin{array}{l}\text { Mainstream und } \\
\text { Alternativmedien } \\
\text { Typ IV }\end{array}$ & 3750 & 52 & 3802 & 0,313 & 802856,091 & 0,343 \\
\hline heiseonline & $\begin{array}{l}\text { Mainstream und } \\
\text { Alternativmedien } \\
\text { Typ IV }\end{array}$ & 3715 & 4 & 3719 & 0,259 & 350364,891 & 0,299 \\
\hline $\operatorname{CDU}$ & $\begin{array}{l}\text { Mainstream und } \\
\text { Alternativmedien } \\
\text { Typ IV }\end{array}$ & 3445 & 109 & 3554 & 0,323 & 2212217,949 & 0,408 \\
\hline phoenix_de & $\begin{array}{l}\text { Mainstream und } \\
\text { Alternativmedien } \\
\text { Typ IV }\end{array}$ & 3422 & 114 & 3536 & 0,324 & 1550842,256 & 0,381 \\
\hline florianklenk & $\begin{array}{l}\text { Mainstream und } \\
\text { Alternativmedien } \\
\text { Typ IV }\end{array}$ & 3212 & 186 & 3398 & 0,331 & 2500199,405 & 0,314 \\
\hline krautreporter & $\begin{array}{l}\text { Mainstream und } \\
\text { Alternativmedien } \\
\text { Typ IV }\end{array}$ & 3180 & 316 & 3496 & 0,352 & 6094801,403 & 0,295 \\
\hline handelsblatt & $\begin{array}{l}\text { Mainstream und } \\
\text { Alternativmedien } \\
\text { Typ IV }\end{array}$ & 3033 & 74 & 3107 & 0,323 & 2231336,339 & 0,328 \\
\hline
\end{tabular}




\begin{tabular}{|c|c|c|c|c|c|c|c|}
\hline Account & Modularity Class & $\begin{array}{c}\text { In- } \\
\text { degree }\end{array}$ & $\begin{array}{c}\text { Out- } \\
\text { degree }\end{array}$ & Degree & $\begin{array}{l}\text { Closeness } \\
\text { Centrality }\end{array}$ & $\begin{array}{c}\text { Betweenness } \\
\text { Centrality }\end{array}$ & $\begin{array}{c}\text { Eigen. } \\
\text { Centrality }\end{array}$ \\
\hline heutejournal & $\begin{array}{l}\text { Mainstream und } \\
\text { Alternativmedien } \\
\text { Typ IV }\end{array}$ & 2999 & 24 & 3023 & 0,292 & 418705,982 & 0,295 \\
\hline ndaktuell & $\begin{array}{l}\text { Mainstream und } \\
\text { Alternativmedien } \\
\text { Typ IV }\end{array}$ & 2965 & 139 & 3104 & 0,330 & 1443712,460 & 0,273 \\
\hline NZZ & $\begin{array}{l}\text { Schweiz- } \\
\text { Community }\end{array}$ & 5535 & 425 & 5960 & 0,351 & 13396622,055 & 0,706 \\
\hline $\begin{array}{l}\text { Republik } \\
\text { Magazin }\end{array}$ & $\begin{array}{l}\text { Schweiz- } \\
\text { Community }\end{array}$ & 1605 & 65 & 1670 & 0,308 & 463831,430 & 0,138 \\
\hline tagesanzeiger & $\begin{array}{l}\text { Schweiz- } \\
\text { Community }\end{array}$ & 1428 & 227 & 1655 & 0,335 & 1387079,077 & 0,158 \\
\hline Weltwoche & $\begin{array}{l}\text { Schweiz- } \\
\text { Community }\end{array}$ & 1222 & 125 & 1347 & 0,335 & 1373119,551 & 0,184 \\
\hline $\begin{array}{l}\text { operationli- } \\
\text { bero }\end{array}$ & $\begin{array}{l}\text { Schweiz- } \\
\text { Community }\end{array}$ & 992 & 580 & 1572 & 0,353 & 4259646,683 & 0,076 \\
\hline adfichter & $\begin{array}{l}\text { Schweiz- } \\
\text { Community }\end{array}$ & 966 & 379 & 1345 & 0,352 & 1491068,246 & 0,103 \\
\hline DBinswanger & $\begin{array}{l}\text { Schweiz- } \\
\text { Community }\end{array}$ & 703 & 46 & 749 & 0,297 & 97251,057 & 0,059 \\
\hline spschweiz & $\begin{array}{l}\text { Schweiz- } \\
\text { Community }\end{array}$ & 696 & 159 & 855 & 0,322 & 250103,059 & 0,048 \\
\hline Knutti_ETH & $\begin{array}{l}\text { Schweiz- } \\
\text { Community }\end{array}$ & 663 & 79 & 742 & 0,314 & 471236,220 & 0,048 \\
\hline GrueneCH & $\begin{array}{l}\text { Schweiz- } \\
\text { Community }\end{array}$ & 657 & 190 & 847 & 0,319 & 378828,012 & 0,049 \\
\hline RolandTichy & $\begin{array}{l}\text { Alternativmedien } \\
\text { Typ I, II }\end{array}$ & 6082 & 148 & 6230 & 0,349 & 6199143,905 & 1,000 \\
\hline TichysEinblick & $\begin{array}{l}\text { Alternativmedien } \\
\text { Typ } 1, \|\end{array}$ & 5268 & 41 & 5309 & 0,309 & 1061993,768 & 0,839 \\
\hline Steinhoefel & $\begin{array}{l}\text { Alternativmedien } \\
\text { Typ I, II }\end{array}$ & 5197 & 50 & 5247 & 0,320 & 722893,055 & 0,924 \\
\hline AfD & $\begin{array}{l}\text { Alternativmedien } \\
\text { Typ I, II }\end{array}$ & 5115 & 270 & 5385 & 0,351 & 8940149,257 & 0,846 \\
\hline Achgut_com & $\begin{array}{l}\text { Alternativmedien } \\
\text { Typ I, II }\end{array}$ & 4747 & 102 & 4849 & 0,336 & 2321319,797 & 0,818 \\
\hline $\begin{array}{l}\text { SteinbachE- } \\
\text { rika }\end{array}$ & $\begin{array}{l}\text { Alternativmedien } \\
\text { Typ I, II }\end{array}$ & 4475 & 52 & 4527 & 0,334 & 1191490,268 & 0,833 \\
\hline Junge_Freiheit & $\begin{array}{l}\text { Alternativmedien } \\
\text { Typ } 1, \| \\
\end{array}$ & 4470 & 128 & 4598 & 0,343 & 2347104,641 & 0,789 \\
\hline $\begin{array}{l}\text { Beatrix_- } \\
\text { vStorch }\end{array}$ & $\begin{array}{l}\text { Alternativmedien } \\
\text { Typ I, II }\end{array}$ & 4413 & 236 & 4649 & 0,356 & 3763384,463 & 0,786 \\
\hline Hartes_Geld & $\begin{array}{l}\text { Alternativmedien } \\
\text { Typ I, II }\end{array}$ & 3800 & 260 & 4060 & 0,359 & 2968994,225 & 0,770 \\
\hline
\end{tabular}




\begin{tabular}{|c|c|c|c|c|c|c|c|}
\hline Account & Modularity Class & $\begin{array}{c}\text { In- } \\
\text { degree }\end{array}$ & $\begin{array}{c}\text { Out- } \\
\text { degree }\end{array}$ & Degree & $\begin{array}{l}\text { Closeness } \\
\text { Centrality } \\
\end{array}$ & $\begin{array}{c}\text { Betweenness } \\
\text { Centrality }\end{array}$ & $\begin{array}{c}\text { Eigen. } \\
\text { Centrality }\end{array}$ \\
\hline RT_Deutsch & $\begin{array}{l}\text { Alternativmedien } \\
\text { Typ I, II }\end{array}$ & 3787 & 64 & 3851 & 0,330 & 5373742,471 & 0,538 \\
\hline $\begin{array}{l}\text { COMPACT } \\
\text { Magazin }\end{array}$ & $\begin{array}{l}\text { Alternativmedien } \\
\text { Typ I, II }\end{array}$ & 3719 & 967 & 4686 & 0,382 & 15223225,551 & 0,771 \\
\hline cicero_online & $\begin{array}{l}\text { Alternativmedien } \\
\text { Typ I, II }\end{array}$ & 3563 & 186 & 3749 & 0,348 & 5249215,338 & 0,514 \\
\hline BjoernHoecke & $\begin{array}{l}\text { Alternativmedien } \\
\text { Typ I, II }\end{array}$ & 3239 & 65 & 3304 & 0,315 & 870247,145 & 0,579 \\
\hline IBDeutschland & $\begin{array}{l}\text { Alternativmedien } \\
\text { Typ I, II }\end{array}$ & 3100 & 57 & 3157 & 0,306 & 351725,785 & 0,575 \\
\hline Martin_Sellner & $\begin{array}{l}\text { Alternativmedien } \\
\text { Typ I, II }\end{array}$ & 3099 & 574 & 3673 & 0,374 & 6652927,843 & 0,623 \\
\hline KoeppelRoger & $\begin{array}{l}\text { Alternativmedien } \\
\text { Typ I, II }\end{array}$ & 3061 & 37 & 3098 & 0,310 & 510154,721 & 0,601 \\
\hline Einzelfallinfos & $\begin{array}{l}\text { Alternativmedien } \\
\text { Typ I, II }\end{array}$ & 3008 & 0 & 3008 & 0,000 & 0,000 & 0,653 \\
\hline AfDKompakt & $\begin{array}{l}\text { Alternativmedien } \\
\text { Typ I, II }\end{array}$ & 2880 & 281 & 3161 & 0,352 & 2580645,972 & 0,597 \\
\hline de_sputnik & $\begin{array}{l}\text { Alternativmedien } \\
\text { Typ I, II }\end{array}$ & 2392 & 26 & 2418 & 0,306 & 821193,906 & 0,356 \\
\hline $\begin{array}{l}\text { Netzdenun- } \\
\text { ziant }\end{array}$ & $\begin{array}{l}\text { Alternativmedien } \\
\text { Typ I, II }\end{array}$ & 2353 & 1838 & 4191 & 0,397 & 2704686,173 & 0,682 \\
\hline ein_prozent & $\begin{array}{l}\text { Alternativmedien } \\
\text { Typ I, II }\end{array}$ & 2346 & 427 & 2773 & 0,365 & 2463004,035 & 0,504 \\
\hline DanieleGanser & $\begin{array}{l}\text { Alternativmedien } \\
\text { Typ I, II }\end{array}$ & 2116 & 2 & 2118 & 0,258 & 245461,036 & 0,228 \\
\hline Zeitgeschehen_ & $\begin{array}{l}\text { Alternativmedien } \\
\text { Typ I, II }\end{array}$ & 2055 & 2046 & 4101 & 0,411 & 9177523,682 & 0,575 \\
\hline deutsch365 & $\begin{array}{l}\text { Alternativmedien } \\
\text { Typ I, II }\end{array}$ & 1777 & 0 & 1777 & 0,000 & 0,000 & 0,453 \\
\hline jouwatch & $\begin{array}{l}\text { Alternativmedien } \\
\text { Typ I, ॥ }\end{array}$ & 1746 & 111 & 1857 & 0,353 & 1223067,097 & 0,425 \\
\hline cashkurs & $\begin{array}{l}\text { Alternativmedien } \\
\text { Typ I, II }\end{array}$ & 1730 & 1 & 1731 & 0,238 & 244281,419 & 0,255 \\
\hline
\end{tabular}

